\title{
Assosiation of Epicardial and Pericardial Fat Thickness with Coronary Artery Disease
}

Fereshteh Ghaderi ${ }^{1}$, Ali Eshraghi ${ }^{1}$, Alireza Sepehri Shamloo ${ }^{2}$, Sareh Mousavi ${ }^{3}$

\author{
${ }^{1}$ M.D., Assistant Professor, Department of Cardiology, Atherosclerosis Prevention Research Center, Imam Reza \\ Hospital, Faculty of Medicine, Mashhad University of Medical Sciences, Mashhad, Iran \\ ${ }^{2}$ M.D., Research Administrator, Department of Cardiac Surgery, Faculty of Medicine, Imam Reza Hospital, \\ Mashhad University of Medical Sciences, Mashhad, Iran \\ ${ }^{3}$ M.D., Cardiologist, Department of Cardiology, Faculty of Medicine, Imam Reza Hospital, Mashhad University of \\ Medical Sciences, Mashhad, Iran
}

\section{Type of article: Original}

\begin{abstract}
Introduction: Visceral adipose tissue is a known important risk factor for coronary artery disease (CAD). While some studies have suggested relationship between epicardial fat thickness (EFT) and CAD, there are no adequate studies for pericardial fat thickness (PFT). The aim of this study was to determine the association of EFT and PFT with CAD.

Methods: This cross-sectional study was conducted on patients who were candidates for elective coronary artery angiography, referred to Emam Reza Hospital, Mashhad, Iran during Jan 2014-2016. Demographic and laboratory data were collected. Transthoracic echocardiography was performed to determine average EFT and PFT at the standard parasternal long-axis view at end-systole for 3 cardiac cycles. SCA was performed on the same day. The patients were divided into two groups: CAD $(n=59)$ and non-CAD $(n=41)$ based on presence or absence of epicardial coronary artery stenosis of $>50 \%$. Chi-square, independent T-test, and receiver operating characteristic (ROC) curve were used by SPSS Version 16 for data analysis.

Results: One hundred patients ( 44 women and 56 men) with an average age of $56.4 \pm 9.9$ years were studied. The two groups were not significantly different in demographic profile and cronary risk factors. While PFT was not significantly different between the two groups, EFT was significantly higher in CAD group $(3.0 \pm 3.69$ vs. $1.2 \pm$ $3.6, \mathrm{p}<0.0001)$. Moreover, with the increase of the affected coronary arteries, EFT increased $(\mathrm{p}<0.0001)$. Gensini score had a strong correlation with amount of EFT $(r=0.765, p<0.0001)$. EFT with a cutoff value of $4.25 \mathrm{~mm}$ (sensitivity $=79 \%$, specificity $=68 \%$ ) was specified in predicting CAD.

Conclusion: EFT measured by echocardiography can be used as an independent marker to predict CAD. More studies are needed to determine the predictive role of PFT for CAD.

Keywords: Epicardial fat thickness, Pericardial fat thickness, Coronary artery disease, Echocardiography
\end{abstract}

\section{Introduction}

Visceral adipose tissue is regarded as an important risk factor in the development of metabolic syndrome and Coronary Artery Disease (CAD) (4-2). The anatomical and functional proximity of the myocardium to the visceral adipose tissue around it, as well as the metabolic activity of this tissue are two of the reasons that have increased the importance of adipose tissue around the heart in recent years (5). It seems that visceral adipose tissue around the heart is contributing to the development of coronary atherosclerosis due to inflammatory and secretory mechanisms $(8-6,4)$ Echocardiography is a safe and relatively cheap and easy way to evaluate the amount of fat around the heart (5). The evidence shows that echocardiographic Epicardial Fat Thickness (EFT) significantly increased in patients with CAD, and this is significantly associated with the severity and extent of coronary artery involvement (9-18). But pericardial fat thickness (PFT) is another visceral adipose tissue around the heart, and the evidence is very

\section{Corresponding author:}

Dr. Sareh Mousavi, Cardiologist, Department of Cardiology, Faculty of Medicine, Imam Reza Hospital, Mashhad University of Medical Sciences, Mashhad, Iran. Tel: +98.5138544504, Email: moosavi_sa@yahoo.com

Received: March 06, 2016, Accepted: June 01, 2016, Published: September 2016

iThenticate screening: June 01, 2016, English editing: August 02, 2016, Quality control: September 04, 2016

(C) 2016 The Authors. This is an open access article under the terms of the Creative Commons Attribution-NonCommercialNoDerivs License, which permits use and distribution in any medium, provided the original work is properly cited, the use is non-commercial and no modifications or adaptations are made. 
limited about its association with the development of atherosclerosis (1). If we can find out the existence of CAD using status of visceral fat around the heart of the noninvasive echocardiography method, unnecessary invasive angiographies can be prevented in future. However, more evidence is needed for the introduction of this method as a routine way to predict CAD. But, very few studies have been conducted to determine the optimal cutoff for predicting CAD based on visceral fat thickness around the heart. Thus, this study aims at investigating the relationship between Epicardial and PFT with severity and extent of coronary artery involvement.

\section{Material and Methods}

\subsection{Study Design and sampling}

This cross-sectional study was conducted on patients who were candidates for elective coronary angiography based on history, ECG and/or stress tests, referred to Emam Reza Hospital, Mashhad, Iran during Jan 2014-2016. The sample size was calculated according to Ho Yun et al. using w=1/2 Ln 1+r/1-r with taking into consideration $\alpha=$ 0.05 and $\beta=0.2$ for each group of 50 patients. Non-probability purposive sampling was used to select samples. We used this sampling method because there was an interest of the research in a specific field, which was patients with SCA.

\subsection{Selection criteria}

Inclusion criteria consisted of patients who were candidates forelective angiography, and who were admitted to the hospital, and gave consent to participate in the study. Exclusion criteria were: patients with heart valve stenosis, systolic heart failure as the ventricular ejection fraction below 50\%, Pericardial disease, body mass index (BMI) greater than $40 \mathrm{~kg} / \mathrm{m}^{2}$, systemic disease (except those known as coronary risk factors), and patients with suboptimal echocardiographic images

\subsection{Data collection}

Demographic data, including age, sex, weight, height, abdominal circumference, hip size, BMI and waist-to-hip ratio were collected. Also, if the patients had experienced any of: risk factors for diabetes, hypertension, or smoking, their positive record was registered into the questionnaire. Patients underwent angiography and were divided into two groups of normal - and patients regarding CAD, based on the presence of significant (stenosis greater than 50\%) or insignificant coronary artery obstruction. After angiography, all patients underwent echocardiography regarding determining Epicardial and Pericardial Fat Thickness.

\subsection{Echocardiography}

Echocardiography was performed by a cardiologist. All echocardiography tests were performed by an echocardiography device with frequency probe (3-5 HZ) (Vivid 3, GE, USA). EFT was measured as a line perpendicular to the free wall of the right ventricle (the vertical line of point of the wall in the middle of view and aortic annulus) in the left lateral position at the standard parasternal long-axis view. EFT was measured as the maximum size at the end of systole in 3 cardiac cycles, and the average of the three measurements was evaluated as EFT. The same procedure was repeated to determine the pericardial fat thickness.

\subsection{Selective coronary Angiography}

A Siemens Artis (Model 2012) was used to do angiography tests. CAD was specified as stenosis greater than $20 \%$ in all CADs. Stenosis ranging from $20 \%-50 \%$ was specified as minimal CAD; Stenosis greater than $50 \%$ was specified as obstructive CAD. Otherwise, it was defined as normal coronary arteries. Coronary angiograms were defined by two independent invasive cardiologists. Gensini score for severity of CAD was determined for all patients.

\subsection{Statistical analysis}

Demographical and paraclinical data of patients were entered into a computer and analyzed using SPSS 17.0 for Windows (SSPS Inc., Chicago, IL, USA). Data are expressed as mean \pm SD and $\mathrm{p}<0.05$ is considered significant. To analyze the quantitative variables, the t-test was used, and if these variables were not normal, the Mann-Whitney test was used. To analyze qualitative variables, Pearson's chi-squared test was used, and if necessary, Fisher's exact test was used. To determine the difference between the values of a variable among several independent (statistical) populations, the ANOVA test or its non-parametric equivalent, Kruskal-Wallis was used, and to evaluate the variables at the same time for prognosis of the disease, a multiple logistic regression model was used. Moreover, to determine the cutoff point, Epicardial and PFT were used for predicting CAD from ROC curve. 
http://www.ephysician.ir

\subsection{Ethical considerations}

The study was approved by the Ethics Committee of Mashhad University of Medical Sciences (Code: 910764). Informed consent was obtained from all Voluntary participants, before their inclusion in the study.

\section{Results}

\subsection{Baseline characteristics}

During a year of study, 118 patients who were candidates for angiography were enrolled, among which 18 patients were excluded due to exclusion criteria. Three patients, due to abnormal valve narrowing (stenosis), five patients due to systolic heart failure, three patients due to pericardial effusion or constrictive pericarditis, four patients for having a Body Mass Index (BMI) greater than 40 and three patients due to medical illness were excluded from the study. In this study, 100 patients (56 men) with a mean age of $56.4 \pm 9.9$ years were studied in the two groups (59 patients having coronary artery stenosis of more than $50 \%=\mathrm{CAD}$ group, and 41 patients without CAD or with CAD below $50 \%$ stenosis $=$ Non-CAD group). The two groups were not significantly different regarding demographic data and risk factors of diabetes, smoking, high blood pressure, Dyslipidemia (Table 1).

Table 1. Demographic data in study population, with and without obstructive coronary artery disease (CAD)

\begin{tabular}{|l|l|l|l|}
\hline Variable & Non-CAD group, $(\mathrm{n}=41)$ & CAD group, $(\mathrm{n}=59)$ & $\mathrm{p}$-value \\
\hline Male, $\mathrm{n}(\%)$ & $25(61)$ & $31(52.5)$ & 0.403 \\
\hline Age, mean \pm SD (years) & $57.2 \pm 7.3$ & $59.8 \pm 9.1$ & 0.258 \\
\hline Diabetes, $\mathrm{n}(\%)$ & $5(12.5)$ & $12(20.7)$ & 0.293 \\
\hline Smoking, $\mathrm{n}(\%)$ & $12(29.2)$ & $20(33.9)$ & 0.652 \\
\hline Hypertension, $\mathrm{n}(\%)$ & $21(51.2)$ & $30(50.8)$ & 0.971 \\
\hline Dyslipidemia, $\mathrm{n}(\%)$ & $13(31.7)$ & $26(44.8)$ & 0.188 \\
\hline Waist-to-hip ratio, mean \pm SD & $0.9 \pm 0.06$ & $0.9 \pm 0.06$ & 0.717 \\
\hline Waist Circumference $(\mathrm{cm})$, mean \pm SD & $98.2 \pm 11.1$ & $99.2 \pm 11.3$ & 0.842 \\
\hline Body Mass Index $\left(\left(\mathrm{kg} / \mathrm{m}^{2}\right)\right)$, mean \pm SD & $26.4 \pm 4.6$ & $26.8 \pm 4.7$ & 0.798 \\
\hline Epicardial Fat Thickness $(\mathrm{mm})$, mean \pm SD & $3.6 \pm 1.2$ & $6.9 \pm 3.0$ & 0.00 \\
\hline Pericardial Fat Thickness (mm), mean \pm SD & $3.0 \pm 6.2$ & $5.7 \pm 2.8$ & 0.863 \\
\hline Gensini Score, mean \pm SD & $5.2 \pm 4.3$ & $29.4 \pm 17.8$ & 0.00 \\
\hline
\end{tabular}

\subsection{Association of EFT and PFT with presence and severity of CAD}

Average EFT and PFT in the samples under study was $5.3 \pm 2.8$ and $5.1 \pm 2.9 \mathrm{~mm}$ respectively. EFT was significantly higher in the CAD group than the Non-CAD group ( $3.0 \pm 6.9$ versus $1.2 \pm 3.6 \mathrm{~mm}, \mathrm{p}<0.0001)$, but the two groups showed no significant difference regarding PFT (Table 1). Moreover, with the increase of the affected coronary arteries, EFT increased $(\mathrm{p}<0.0001)$ while, the pericardial fat thickness, was not directly related to the number of affected coronary arteries. The Gensini score had a strong significant correlation with the amount of EFT $(\mathrm{r}=0.765, \mathrm{p}<0.0001)$; however, a significant correlation was not found between PFT and the Gensini score $(\mathrm{r}=$ $0.058, \mathrm{p}=0.566$ ). ROC curve was used to find the cutoff point for Variable Epicardial Fat Thickness. Based on the results of the ROC curve, the best cutoff point for the CAD is based on the EFT and was calculated as $4.25 \mathrm{~mm}$ $(95 \%$ CI $[0.740-0.904], \mathrm{p}<0.001$, AROC $=0.82)$. Sensitivity and specificity of this point in the differentiation of $\mathrm{CAD}$ are $79 \%$ and $68 \%$, respectively (Figure 1 ).

\subsection{Relation between epicardial-pericardial fat thickness and clinical variables}

This study showed that EFT was associated with dyslipidemia ( $\mathrm{p}=0.10,95 \%$ CI [0.174-1.256]), however PFT was related to dyslipidemia (95\% CI [0.0174-1.256], $\mathrm{p}<0.0001)$, hypertension $(95 \% \mathrm{CI}$ [1.125-2.394], $\mathrm{p}<0.0001)$ and smoking (95\% CI [-0.556--0.497], $\mathrm{p}=0.007)$. Moreover, there was weak correlation between epicardial fat thickness and BMI or abdominal circumference $(r=0.260, p=0.009$ and $r=0.256, p=0.008)$. Our findings also, showed weak correlation between PFT and BMI $(r=0.282, p=0.004$ and $r=0.318, p=0.001$, respectively) (Table 2). Furthermore, there was no correlation between PFT and EFT with gender, age or waist-to-hip ratio. 


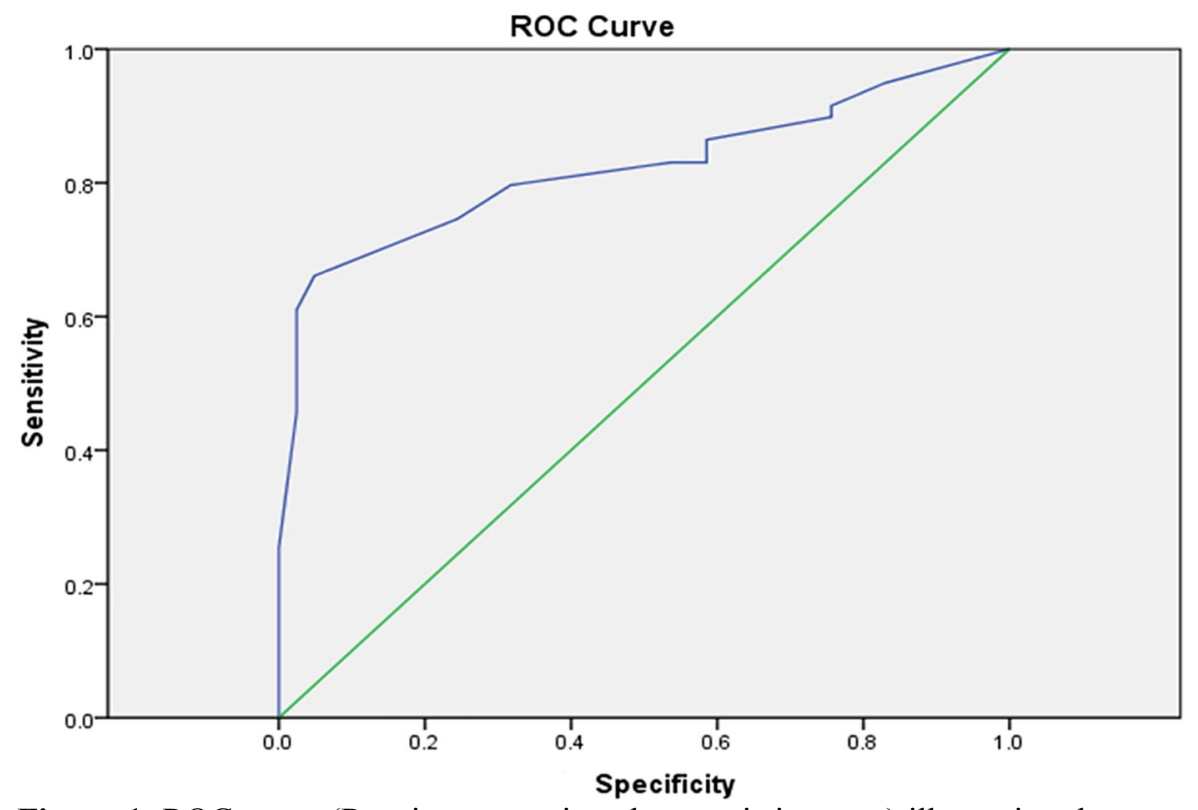

Figure 1. ROC curve (Receiver operating characteristic curve) illustrating the accuracy of Epicardial Fat Thickness for predicting coronary artery disease

Table 2. Correlation of clinical quantitative variables with Epicardial and Pericardial Fat Thickness

\begin{tabular}{|l|l|l|l|l|}
\hline \multirow{2}{*}{ Variables } & \multicolumn{2}{|l|}{ Epicardial Fat Thickness } & \multicolumn{2}{l|}{ Pericardial Fat Thickness } \\
\cline { 2 - 5 } & $95 \%$ CI & p-value & $95 \%$ CI & p-value \\
\hline Age & $-0.816-0.167$ & 0.196 & $-1.410--0.43$ & 0.196 \\
\hline Diabetes & $-1.022-0.247$ & 0.232 & $1.125-2.394$ & 0.001 \\
\hline Hypertension & $-0.086-0.901$ & 0.106 & $-0.566-0.422$ & 0.775 \\
\hline Dyslipidemia & $0.174-1.256$ & 0.010 & $0.174-1.256$ & 0.001 \\
\hline Smoking & $-0.556-0.497$ & 0.913 & $-0.566-0.497$ & 0.007 \\
\hline
\end{tabular}

\section{Discussion}

The aim of this study was to evaluate the predictive role of EFT and PFT with presence and severity of CAD. At first, the results of this study showed that EFT in patients with CAD was significantly higher than the group without CAD while this finding was not observed in the relationship between PFT and CAD. The second finding is the number of involved coronary arteries and severity of obstruction that have a significant relationship with the PFT while this relationship was not observed in the pericardial layer. The third finding is the relationship between PFT and EFT atherosclerosis risk factors in the regression model. According to findings of this study, in EFT model, dyslipidemia risk factor and in Pericardial Fat Thickness, risk factors of dyslipidemia, smoking, and diabetes have been associated with changes in thickness. The fourth major finding also showed that according to the ROC curve, $4.25 \mathrm{~mm}$ cutoff point of EFT is the best location for prognosis of CAD. The process of findings of the relationship between CAD and EFT in past studies was in such a way that today, EFT increase is known as a predictor of the severity of CAD (4). There are two possible mechanisms for the association between EFT and intensity of Atherosclerosis: First, EFT is one of the components of visceral fat and thereby is associated with metabolic syndrome and cardiovascular risk factors $(2,18)$. Second, from another point of view, it is paracrine and endocrine role intended for Epicardial Fat Thickness. Thus, the epicardial fat can secrete several biologically active molecules such as adiponectin, resistin and other inflammatory cytokines such as interleukin IL-6,1b and tumor necrosis factor $(4,19)$. It should be noted that even inflammatory cytokines that have a foreign origin of the coronary arteries, can cause changes in the intimal lining and provide grounds for the atherosclerosis $(4,20,21)$. Also, evidence shows that histological changes, inside or around the epicardial coronary arteries may underlie vascular homeostasis, vascular endothelium dysfunction, strengthening of vascular inflammation, lesions in the intima, and atheromatous plaque formations by outside-to-inside signaling mechanism (6-8). Therefore, given that the incidence of CAD is directly related to the formation of atherosclerotic plaques, and also, atherosclerosis is considered an inflammatory disease, it can be expected that the epicardial fat area plays a major role through its inflammatory function in the exacerbation of artery atherosclerosis and intensification of coronary stenosis $(2,13,14,22)$. 
The findings of this research regarding the significant relationship between EFT with the existence and severity of CAD is also shown in most other studies, and it seems that the fact that increasing EFT is an important factor in the projecting increase in the severity of CAD is well known (16, 17, 23-26). In Gorter, Eroglu, Gil, and Shemirani's studies, a significant positive correlation was found between EFT and the severity of CAD $(14,21,27,28)$. Also, these studies have shown that EFT in patients with CAD was significantly more, compared to those without CAD $(12,14,21,27,28)$. In the meta-analysis studies carried out on 654 patients with CAD and 495 patients without CAD, it was also found that the level of EFT in patients with CAD was significantly higher, and severity of CAD involvement was significantly related to EFT (26). In the present study, for a closer look at the relationship between EFT and severity of CAD involvement, the quantitative angiographic Gensini Score was used to express the severity of atherosclerotic involvement, based on this index, EFT had a strong linear and significant relationship with the severity of CAD involvement. Thus, this study is excellent regarding using more accurate index Gensini compared to a ranking variable of some arteries with obstructive stenosis used in some studies as the sole atherosclerotic involvement criterion. However, so far at least in 4 other studies, the relationship of EFT has been addressed using scoring systems of severity and extent of coronary artery involvement $(13,14,29)$. However, in three cases of the studies, the quantitative correlation has not been studied between score and Epicardial Fat Thickness. But, the ranking findings of these studies have shown that EFT has significantly increased in higher scores. In another study, a significant correlation was also reported between EFT and the Gensini score $(r=0.600, p<0.001)$ which is consistent with ours. In explaining the reason for the difference between the level of significance of the relationship between EFT and PFT, and the severity of coronary artery involvement, racial and regional differences of people under study resulting in differences in the structure and size of body and fat distribution should also be mentioned. In the European studies conducted by Natale, the thickness $\geq 7 \mathrm{~cm}$ was known as a predictor of CAD and subclinical atherosclerosis. While in another similar study, this value was $5.2 \mathrm{~mm}$ and $3 \mathrm{~mm}$ in a Korean population $(5,11,12$, 30). The role of the tool used to determine the thickness of fat should also be considered because the tools used in the studies were not identical (Ranging from echocardiography, MDCT, and MRI). Moreover, there are technical differences in how to measure tissue thickness with echocardiography between different studies, including measuring thickness in cardiac systole or diastole, used echocardiographic window type, and a location in each window that thickness is measured. Since echocardiography interpretation is ultimately related to the studies, different results can also be achieved by the different interpreters. Other causes of differences in the level of significance of these correlations can be due to sample volume.

Although numerous studies have examined the relationship between EFT and CAD, evidence about the relationship between PFT and severity of coronary artery involvement is very limited. No significant correlation was found in different studies between the PFT and the severity of coronary artery involvement to be consistent with the findings of this study (14); and yet, in another study that applied MRI together with echocardiography, pericardial thickness independent of the used modality was associated with CAD (1). However, despite the obtained findings, the role of PFT cannot be easily ignored in the incidence of CAD. According to the findings of this study, PFT has a significant relationship with the presence or absence of dyslipidemia considered as one of the known atherosclerosis risk factors, so it seems that PFT should be evaluated in further studies. In the present study, EFT was associated with BMI and abdominal circumference, while none of the anthropometric variables showed significant correlation with pericardial fat thickness. Although none of the studies examined the relationship between PFT and anthropometric variables, correlation of EFT has been determined in several studies. Eroglu et al. have shown a significant correlation between EFT and abdominal circumference (2). In an Iacobellis et al. study, a strong correlation was found between BMI and Epicardial Fat Thickness (9). Also, a significant relationship was found between BMI and waist circumference and EFT in several other studies in line with the findings of this study $(2,3,10,12,22)$. However, what is important is that the BMI and abdominal circumference are two factors closely related to visceral fat, and also, as already mentioned, fat tissue out of the coronary arteries can be productive of atherosclerosis through secretion of inflammatory cytokines and other biological factors. Thus, this significant relationship strengthens the importance of EFT as a prognostic factor in the occurrence of CAD $(3,4,13,26)$. In the present study, EFT was more associated with anthropometric factors, while PFT was mostly associated with atherosclerosis risk factors, so that from among four recognized risk factors of diabetes, high blood pressure, dyslipidemia, and smoking, EFT was associated with dyslipidemia and PFT was associated with diabetes and smoking in addition to dyslipidemia. However, in an Eroglu et al. study, identified risk factors of dyslipidemia, diabetes, high blood pressure and smoking were significantly greater in patients with EFT $\geq 5.2 \mathrm{~mm}(2)$. But it seems that the reason for differences between Eroglu's study results and our findings, is investigating the relationship of risk factors with PFT with different statistical analyzes. Since all of these four risk factors are considered as factors affecting the incidence of atherosclerosis, a regression model is used for examining the relationship between EFT and PFT and risk factors 
according to which the results were consistent with Gill et al. (28). In this study, no significant relationship was obtained between known atherosclerosis risk factors and EFT according to the regression model. In this study, echocardiography was used to calculate Epicardial Fat Thickness. According to the findings of the current study and other studies, it seems that we can gradually benefit from EFT as a screening method used in coronary artery occlusive disease. In the present study, EFT has a cutoff value of $4.25 \mathrm{~mm}$, a sensitivity of about $80 \%$ and specificity of about $70 \%$ for prediction of CAD. However, it seems that larger sample size could help find a point with higher sensitivity and specificity, because in other studies with larger sample sizes, the cut-off points found for the existence of CAD according to EFT, had higher sensitivity and specificity. Although the sample size is only one factor to explain the difference in sensitivity and specificity - and even the found cut-off point. What is important is the proximity of the cut-off point found in some studies to the present study. In an Eroglu et al. study, 5.2mm cutoff point with a sensitivity of $85 \%$ and specificity of $81 \%$ (ROC area $0.914, \mathrm{p}<0.001,95 \% \mathrm{CI}[0.86-0.96]$ ) were found for prognosis of CAD. (2) In a Gökdeniz et al. study, EFT cut-off point was carried out by intermediate or high SYNTAX Scores (>22) (31). In Gökdeniz's study, $5 \mathrm{~mm}$ cut-off point with a sensitivity of $77.4 \%$ and specificity of 92.2\% (ROC area $0.851,95 \%$ CI [0.775-0.910]) were found for prediction of intermediate or high SYNTAX Scores $(>22)$. Gil et al. also found 2.4 -mm cutoff point with a sensitivity of $80 \%$ and specificity of $73 \%$ (positive predictive value $57 \%$, and negative predictive value $88 \%$ ) (28). It is clear that the difference of the cut-off point in Gil et al. study is more than the other two studies. But it should be considered that factors like race can underlie the differences between cut off points. Because in various studies conducted by racial difference, average EFT was ranging from $2.2 \mathrm{~mm}$ and $9.5 \mathrm{~mm}(5,11,12,30)$. However, based on the findings of this study and other studies, it seems that the sensitivity of about $80 \%$ in EFT for CAD prediction is a repeated finding. But its specificity that will add the value of this criterion in the prediction of CAD, varies in different studies, and more studies are needed to reach the desired point. One important limitation of the present study was to collect information in a manner that is based on the collected routine medical care; therefore, markers such as interleukin-6, Tumor necrosis factors, or other biologically and active inflammatory factors associated with EFT and PFT have not been studied. It is suggested that in future studies, EFT and PFT ought to be done by 3D-echocardiography. It is also recommended that Cohort's study ought to be conducted, regarding examining the changes of EFT and PFT on patients without coronary artery obstruction, to understand better, about the relationship between risk factors and CAD severity with changes in Epicardial and Pericardial Fat Thickness.

\section{Conclusions}

Our Results show that increased EFT is associated with CAD, and this criterion can be used to predict the occurrence and severity of CAD. On the other hand, although there was no difference between PFT scores obtained from patients with $\mathrm{CAD}$ compared to healthy subjects, considering the significant association of this criterion with dyslipidemia, diabetes and smoking risk factors, more studies are needed to determine the prognostic role of PFT for CAD.

\section{Acknowledgments:}

This paper is the result of the research project of cardiology speciality thesis of Dr. Sareh Mousavi. This study was supported by a grant from the Vice Chancellor for Research of the Mashhad University of Medical Sciences for the research project as a medical student thesis, with approval number of 910764.

\section{Conflict of Interest:}

There is no conflict of interest to be declared.

\section{Authors' contributions:}

All authors contributed to this project and article equally. All authors read and approved the final manuscript.

\section{References:}

1) Sicari R, Sironi AM, Petz R, Frassi F, Chubuchny V, De Marchi D, et al. Pericardial rather than epicardial fat is a cardiometabolic risk marker: an MRI vs echo study. J Am Soc Echocardiogr. 2011; 24(10): 115662. doi: 10.1016/j.echo.2011.06.013. PMID: 21795020.

2) Eroglu S, Sade LE, Yildirir A, Bal U, Ozbicer S, Ozgul AS, et al. Epicardial adipose tissue thickness by echocardiography is a marker for the presence and severity of coronary artery disease. Nutr Metab Cardiovasc Dis. 2009; 19(3): 211-7. doi: 10.1016/j.numecd.2008.05.002. PMID: 18718744.

3) Park JS, Ahn SG, Hwang JW, Lim HS, Choi BJ, Choi SY, et al. Impact of body mass index on the relationship of epicardial adipose tissue to metabolic syndrome and coronary artery disease in an Asian 
population. Cardiovasc Diabetol. 2010; 9: 29. doi: 10.1186/1475-2840-9-29. PMID: 20604967, PMCID: PMC2913996.

4) Sacks HS, Fain JN. Human epicardial fat: what is new and what is missing? Clin Exp Pharmacol Physiol. 2011; 38(12): 879-87. doi: 10.1111/j.1440-1681.2011.05601.x. PMID: 21895738.

5) Iacobellis G, Willens HJ, Barbaro G, Sharma AM. Threshold Values of High - risk Echocardiographic Epicardial Fat Thickness. Obesity (Silver Spring). 2008; 16(4): 887-92. doi: 10.1038/oby.2008.6. PMID: 18379565.

6) Pagano PJ, Clark JK, Cifuentes-Pagano ME, Clark SM, Callis GM, Quinn MT. Localization of a constitutively active, phagocyte-like NADPH oxidase in rabbit aortic adventitia: enhancement by angiotensin II. Proc Natl Acad Sci U S A. 1997; 94(26): 14483-8. PMID: 9405639, PMCID: PMC25029.

7) Romano $M$, Sironi $M$, Toniatti $C$. Chronic treatment with interleukin-1 $\beta$ induces coronary intimal lesions and vasospastic responses in pigs in vivo. Immunity. 1997; 6(3): 315-25.

8) Wang HD, Pagano PJ, Du Y, Cayatte AJ, Quinn MT, Brecher P, et al. Superoxide anion from the adventitia of the rat thoracic aorta inactivates nitric oxide. Circ Res. 1998; 82(7): 810-8. doi: 01.RES.82.7.810. PMID: 9562441.

9) Iacobellis G, Ribaudo MC, Assael F, Vecci E, Tiberti C, Zappaterreno A, et al. Echocardiographic epicardial adipose tissue is related toanthropometric and clinical parameters of metabolic syndrome: a new indicator of cardiovascular risk. J Clin Endocrinol Metab. 2003; 88(11): 5163-8. doi: 10.1210/jc.2003030698. PMID: 14602744.

10) Jeong JW, Jeong MH, Yun KH, Oh SK, Park EM, Kim YK, et al. Echocardiographic epicardial fat thickness and coronary artery disease. Circ J. 2007; 71(4): 536-9. PMID: 17384455.

11) Jeong JW, Jeong MH, Yun KH, Oh SK, Park EM, Kim YK, et al. Echocardiographic epicardial fat thickness and coronary artery disease. Circ J. 2007; 71(4): 536-9. PMID: 17384455.

12) Ahn SG, Lim HS, Joe DY, Kang SJ, Choi BJ, Choi SY, et al. Relationship of epicardial adipose tissue by echocardiography to coronary artery disease. Heart. 2008; 94(3): 7. doi: 10.1136/hrt.2007.118471. PMID: 17923467.

13) Djaberi R, Schuijf JD, van Werkhoven JM, Nucifora G, Jukema JW, Bax JJ. Relation of epicardial adipose tissue to coronary atherosclerosis. The American journal of cardiology. 2008; 102(12): 1602-7. doi: 10.1016/j.amjcard.2008.08.010. PMID: 19064012.

14) Gorter PM, de Vos AM, van der Graaf Y, Stella PR, Doevendans PA, Meijs MF, et al. Relation of epicardial and pericoronary fat to coronary atherosclerosis and coronary artery calcium in patients undergoing coronary angiography. Am J Cardiol. 2008; 102(4): 380-5. doi: 10.1016/j.amjcard.2008.04.002. PMID: 18678291.

15) Sade LE, Eroglu S, Bozbaş H, Özbiçer S, Hayran M, Haberal A, et al. Relation between epicardial fat thickness and coronary flow reserve in women with chest pain and angiographically normal coronary arteries. Atherosclerosis. 2009; 204(2): 580-5. doi: 10.1016/j.atherosclerosis.2008.09.038. PMID: 19019370.

16) Bastarrika G, Broncano J, Schoepf UJ, Schwarz F, Lee YS, Abro JA, et al. Relationship between coronary artery disease and epicardial adipose tissue quantification at cardiac CT: comparison between automatic volumetric measurement and manual bidimensional estimation. Acad Radiol. 2010; 17(6): 727-34. doi: 10.1016/j.acra.2010.01.015. PMID: 20363161.

17) Nabati M, Saffar N, Yazdani J, Parsaee MS. Relationship between epicardial fat measured by echocardiography and coronary atherosclerosis: a single-blind historical cohort study. Echocardiography. 2013; 30(5): 505-11. doi: 10.1111/echo.12083. PMID: 23305488.

18) Bachar GN, Dicker D, Kornowski R, Atar E. Epicardial adipose tissue as a predictor of coronary artery disease in asymptomatic subjects. Am J Cardiol. 2012; 110(4): 534-8. doi: 10.1016/j.amjcard.2012.04.024. PMID: 22579086.

19) Toczylowski K, Gruca M, Baranowski M. [Epicardial adipose tissue and its role in cardiac physiology and disease]. Postepy Hig Med Dosw (Online). 2013; 67: 584-93. doi: 10.5604/17322693.1053908. PMID: 23799402.

20) Nelson MR, Mookadam F, Thota V, Emani U, Al Harthi M, Lester SJ, et al. Epicardial fat: an additional measurement for subclinical atherosclerosis and cardiovascular risk stratification? J Am Soc Echocardiogr. 2011; 24(3): 339-45. doi: 10.1016/j.echo.2010.11.008. PMID: 21185148.

21) Shemirani H, Khoshavi M. Correlation of echocardiographic epicardial fat thickness with severity of coronary artery disease-an observational study. Anadolu Kardiyol Derg. 2012; 12(3): 200-5. doi: 10.5152/akd.2012.061. PMID: 22366102. 
22) Gokdeniz T, Turan T, Aykan AC, Gul I, Boyaci F, Hatem E, et al. Relation of epicardial fat thickness and cardio-ankle vascular index to complexity of coronary artery disease in nondiabetic patients. Cardiology. 2013; 124(1): 41-8. doi: 10.1159/000345298. PMID: 23328069.

23) Mustelier JV, Rego JO, González AG, Sarmiento JC, Riverón BV. Echocardiographic parameters of epicardial fat deposition and its relation to coronary artery disease. Arq Bras Cardiol. 2011; 97(2): 122-9. doi: S0066-782X2011005000068. PMID: 21655877.

24) Yorgun H, Canpolat U, Hazırolan T, Sunman H, Ateş AH, Gürses KM, et al. Epicardial adipose tissue thickness predicts descending thoracic aorta atherosclerosis shown by multidetector computed tomography. Int J Cardiovasc Imaging. 2012; 28(4): 911-9. doi: 10.1007/s10554-011-9899-x. PMID: 21637979.

25) Iacobellis G, Lonn E, Lamy A, Singh N, Sharma AM. Epicardial fat thickness and coronary artery disease correlate independently of obesity. Int J Cardiol. 2011; 146(3): 452-4. doi: 10.1016/j.ijcard.2010.10.117. PMID: 21094545.

26) $\mathrm{Xu} \mathrm{Y}$, Cheng $\mathrm{X}$, Hong $\mathrm{K}$, Huang $\mathrm{C}$, Wan $\mathrm{L}$. How to interpret epicardial adipose tissue as a cause of coronary artery disease: a meta-analysis. Coron Artery Dis. 2012; 23(4): 227-33. doi: 10.1097/MCA.0b013e328351ab2c. PMID: 22361934.

27) de Vos AM, Prokop M, Roos CJ, Meijs MF, van der Schouw YT, Rutten A, et al. Peri-coronary epicardial adipose tissue is related to cardiovascular risk factors and coronary artery calcification in post-menopausal women. Eur Heart J. 2008; 29(6): 777-83. doi: 10.1093/eurheartj/ehm564. PMID: 18156138.

28) Bachar GN, Dicker D, Kornowski R, Atar E. Epicardial adipose tissue as a predictor of coronary artery disease in asymptomatic subjects. Am J Cardiol. 2012; 110(4): 534-8. doi: 10.1016/j.amjcard.2012.04.024. PMID: 22579086.

29) Iwasaki K, Matsumoto T, Aono H, Furukawa H, Samukawa M. Relationship Between Epicardial Fat Measured by 64 - Multidetector Computed Tomography and Coronary Artery Disease. Clin Cardiol. 2011; 34(3): 166-71. doi: 10.1002/clc.20840. PMID: 21337349.

30) Chaowalit N, Somers VK, Pellikka PA, Rihal CS, Lopez-Jimenez F. Subepicardial adipose tissue and the presence and severity of coronary artery disease. Atherosclerosis. 2006; 186(2): 354-9. doi: 10.1016/j.atherosclerosis.2005.08.004. PMID: 16183065. 